8. K. A. Mauritz and C. L. Gray, Macromolecules, 16, 1279 (1983).

9. T. Asawa, J. Appl. Electrochem., 19, 566 (1989).

10. D. L. Peet and J. H. Austin, Paper presented at the Chlorine Institute Meeting, Feb. 5, 1986; reprint available from the Du Pont Company, Wilmington, DE.

11. M. Seko, S. Ogawa, H. Ono, and O. Suzuki, in "Advances in the Chlor-Alkali and Chlorate Industry," M. M. Silver and E. M. Spore, Editors, PV 84-11, pp. 49-72, The Electrochemical Society Softbound Proceedings Series, Pennington, NJ (1984).

12. W. G. F. Grot, Abstract 894, p. 1258, The Electrochemical Society Extended Abstracts, Vol. 87-2, Honolulu, HI, Oct. 18-23 (1987).

13. H. L. Yeager, J. D. Malinsky, and R. L. Dotson, in "Transport Processes in Electrochemical Systems," R. S. Yeo, T. Katan, and D.-T. Chin, Editors, PV 82-10, pp. 215-222, The Electrochemical Society Softbound Proceedings Series, Pennington, NJ (1982).

14. H. L. Yeager and J. D. Malinsky, U.S. Pat. 4,545,863 (1985).

15. H. L. Yeager and J. D. Malinsky, in "Coulombic Interactions in Macromolecular Systems," A. Eisenberg and F. E. Bailey, Editors, ACS Symposium Series
No. 302, Chap. 11, American Chemical Society (1986).

16. L. I. Krishtalik, Soviet Electrochem., 15, 632 (1979).

17. A. M. Peers, Disc. Faraday Soc., 21, 124 (1956).

18. H. Reiss and I. Bassignana, J. Mem. Sci., 11, 219 (1982).

19. C. W. Carr and K. Sollner, J. Gen. Physiol., 28, 119 (1944).

20. F. Helfferich, "Ion Exchange," p. 134, McGraw-Hill, Inc., New York (1961)

21. J. T. Keating, Du Pont Company, Unpublished results.

22. O. P. Romashin, M. Fioshin, R. Erenburg, E. Ryabov, V. Kubasov, and L. Krishtalik, Soviet Electrochem., 15, $553(1979)$.

23. "Nafion ${ }^{\mathbb{B}}$ 90209 Product Bulletin," Du Pont Company, Polymer Products Department, Wilmington, DE (1988).

24. J. T. Keating, Du Pont Company, Private communication.

25. S. Lengyel, J. Giber, G. Beke, and A. Vertes, Acta Chim. Hung. Acad. Sci., 39, 357 (1963).

26. V. P. Troshin and E. V. Zvyagina, Soviet Electrochem., 8,1669 (1972).

27. D. A. Lown and H. R. Thirsk, Trans. Faraday Soc., 67, $132(1971)$.

28. I. N. Maksimova and V. F. Yushkevich, Russ. J. Phys. Chem., 37, 475 (1963).

\title{
The Bipolar Analysis of a Single Sphere in an Electrolytic Cell
}

\author{
Shi-Chern Yen* and Ching-Yih Yao \\ Department of Chemical Engineering, National Taiwan University, Taipei, Taiwan, China
}

\begin{abstract}
The bipolar behavior of a single sphere in an electrolytic cell is investigated in this study. A mathematical model is proposed for two surface conditions: linear polarized kinetics and the Butler-Volmer kinetics. Analytical current and potential distributions are obtained for a reversible reaction and linear polarized kinetics. Semianalytical potential and current distributions for the Butler-Volmer kinetics are also obtained by the boundary collocation method. The current enhancement factor is defined as the ratio of the bipolar current to the applied current, and its value is predicted theoretically for linear polarization. The effective conductivity of a dilute dispersion is also formulated and related to the first coefficient $D_{1}$ of the series expression of the potential in a continuous medium. The influences of various parameters, including dimensionless potential drop, $\Psi$, conductivity ratio of electrolyte to solid phase, $\alpha$, ratio of exchange current density to applied current, $\beta$, and transfer coefficients, $\alpha_{a}$ and $\alpha_{c}$, are respectively discussed. The suitability of assuming linear polarized kinetics is also evaluated by comparing it with the Butler-Volmer kinetics.
\end{abstract}

When an electric potential is applied across an electroconductive object that is isolated between the two current feeders, bipolar behavior may occur at the surface of this object, and both anodic and cathodic reactions may occur on one and the other side of its surface, respectively. Bipolar behavior has been discussed in many fields, which include the dissolution of nuclear fuel (1), oxide film thickness control (2), and organic synthesis $(3,4)$, etc. In recent years, bipolar particulate electrodes, including packedbed, trickle-bed, and fluidized bed types, have been broadly investigated $(5,6)$. The bipolar behavior is also concerned with the charge-transfer mechanism in fluidized bed electrodes $(7,8)$.

In the literature some theoretical models were developed to predict the dispersion conductivity (9-11) or energy consumption $(1,4)$ in particulate bipolar cells. But either constant current density or linear potential distribution on the electrode surface was assumed in earlier models. As a matter of fact, the current and potential distributions at the electrode surface are nonuniform and dependent on the relative electric conductivity of the solid and electrolyte phases, the resistance of the electrochemical reactions occurring at the electrode surface, the applied electric field, and the mass transport of the reactants. If the primary current distribution is considered, the resistance of the electrochemical reaction and the mass transfer limitation of the reactants can be neglected. The potential distribution of a spherical particle for a reversible reaction

* Electrochemical Society Active Member. had been solved analytically and was used to estimate the effective conductivity of dilute dispersions of spherical particles by Maxwell $(11,12)$. When the secondary current distribution is relevant, the mass-transfer limitation can also be ignored.

Due to the importance of the bipolar behavior in some electrolytic cells, fundamental studies are necessary for a detailed understanding and for the proper design of bipolar cells. In this paper, a mathematical model for the bipolar behavior on a single sphere is proposed in which the primary and secondary current distributions are considered. The effects of particle size, applied electric field, and solid-phase conductivity on the bipolarity of the sphere can be deduced and estimated exactly according to this model. Analytical solutions have been obtained for cases in which the electrochemical reaction is reversible and when dealing with linear polarized kinetics, respectively. The semianalytical solution for the Butler-Volmer kinetics at the surface is also given by using the boundary collocation method (13). The consumption of electric energy due to the presence of the single sphere in a linear potential field is also formulated. The effective conductivity of a dilute dispersion for linear polarized kinetics or the Butler-Volmer kinetics can be estimated from the consumption of electrical energy.

\section{Analysis}

The schematic diagram of the problem considered is depicted in Fig. 1. The potentials in the solid sphere and the electrolyte are described as $\phi_{1}$ and $\phi_{2}$, respectively. The 


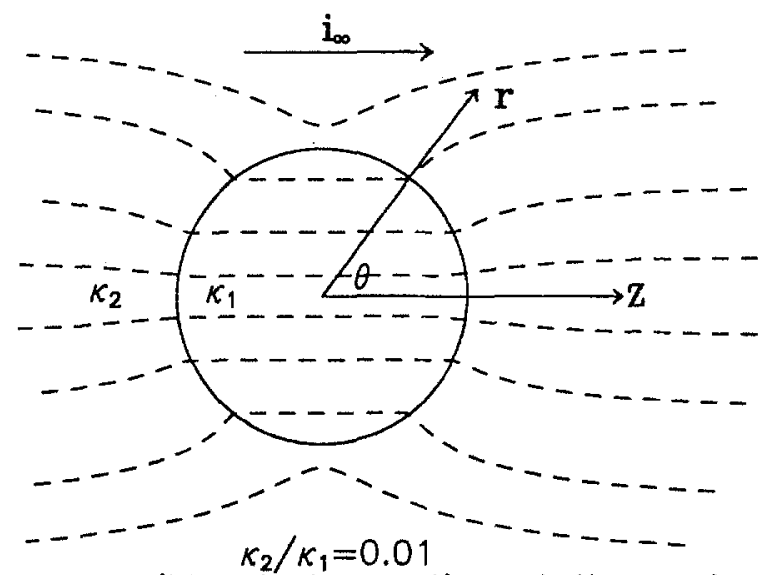

a reversible electroreaction at the surface

Fig. 1. A single sphere in an electrical field.

charge balance equation should be satisfied in each phase, and it can be represented by Laplace's equation with spherical coordinates as follows

$$
\begin{aligned}
& \nabla^{2} \phi_{1}=0 \\
& \nabla^{2} \phi_{2}=0
\end{aligned}
$$

in which

$$
\nabla^{2}=\frac{1}{r^{2}} \frac{\partial}{\partial r}\left(r^{2} \frac{\partial}{\partial r}\right)+\frac{1}{r^{2} \sin \theta} \frac{\partial}{\partial \theta}\left(\sin \theta \frac{\partial}{\partial \theta}\right)
$$

Because only the primary and secondary current distributions are considered, the mass balance equation of the reactant is not involved, and only one electrochemical reaction is assumed to take place at the spherical particle. Then the boundary conditions are expressed as follows: (i) An electrical field is applied, and the potential distribution far from the particle should match the linear potential field; thus, when $r \rightarrow \infty$

$$
\phi_{2}=-\frac{i_{\infty}}{\kappa_{2}} r \cos \theta+\phi_{0}
$$

where $i_{\infty}$ is the applied current density far from the electrode, $\kappa_{2}$ is the electric conductivity of the electrolyte, and $\phi_{0}$ is the reference potential far from the sphere at the plane of $z=0$. (ii) At the center of the sphere $(r=0), \phi_{1}$ is finite. (iii) At the interface between the particle and the electrolyte $(r=R)$, the current densities from the two phases should be equal, due to the flux continuity.Thus, at $r=R$

$$
\kappa_{1} \frac{\partial \phi_{1}}{\partial r}=\kappa_{2} \frac{\partial \phi_{2}}{\partial r}
$$

where $\kappa_{1}$ is the electric conductivity of the particle and $R$ is the radius of the sphere. (iv) The last boundary condition is concerned with the kinetics that involves the electrochemical reaction at the spherical surface $(r=R)$. The electrochemical reaction is considered as one of the following three cases, a reversible reaction, linear polarized kinetics, and kinetics described by the Butler-Volmer equation. The case of Butler-Volmer kinetics is regarded as a general case for an electrode reaction. The electrode kinetics should match the electric current at the interface between the particle and the electrolyte. Therefore, at $r=R$, for a reversible reaction

$$
\phi_{1}-\phi_{2}=E^{\prime}
$$

for linear polarized kinetics

$$
j=i_{0}\left(\frac{n F}{. \not h T}\right)\left(\phi_{1}-\phi_{2}-E^{\prime}\right)=-\left.\kappa_{2} \frac{\partial \phi_{2}}{\partial r}\right|_{r=R}[6]
$$

and for the Butler-Volmer equation

$$
\begin{aligned}
j=i_{0} & \left\{\exp \left[\alpha_{\mathrm{a}} \frac{n F}{\text {.hT }}\left(\phi_{1}-\phi_{2}-E^{\prime}\right)\right]\right. \\
& \left.\quad-\exp \left[-\alpha_{\mathrm{c}} \frac{n F}{\text {. } R T}\left(\phi_{1}-\phi_{2}-E^{\prime}\right)\right]\right\}=-\left.\kappa_{2} \frac{\partial \phi_{2}}{\partial r}\right|_{\tau=R}
\end{aligned}
$$

where $E^{\prime}$ is the Nernst potential of the reaction, $i_{0}$ is the exchange current density, $\alpha_{\mathrm{a}}$ and $\alpha_{\mathrm{c}}$ are anodic and cathodic transfer coefficients, respectively. One of Eq. [5]-[7] represents the boundary condition, which is dependent on the reversibility of the electrode reaction, and Eq. [3]-[4] are the other boundary conditions holding for all three cases.

In order to make the governing equation dimensionless, the following dimensionless variables and parameters are defined

$\Phi_{1}=\frac{\phi_{1}}{\mathrm{~V}}, \quad \Phi_{2}=\frac{\phi_{2}}{\mathrm{~V}}, \quad \Phi_{0}=\frac{\phi_{0}}{\mathrm{~V}}$,

$$
E=\frac{E^{\prime}}{\mathrm{V}}, \quad \xi=\frac{r}{R}
$$

and

$$
\alpha=\frac{\kappa_{2}}{\kappa_{1}}, \quad \beta=\frac{i_{0}}{i_{\infty}}, \quad \Psi=\frac{n F}{\mathscr{R} T} V
$$

where $V=i_{\infty} R / \kappa_{2}$. The parameter $\alpha$ represents the relative electric conductivity of the electrolyte to the solid electrode, and if $\alpha \rightarrow \infty$, the sphere can be considered as insulated; if $\alpha \rightarrow 0$, the potential in the sphere can be regarded as uniform. The parameter $\beta$ is the ratio of exchange current density to the applied current density; larger $\beta$ means that the electrochemical reaction tends to approach complete reversibility. Smaller $\beta$ indicates that the exchange current density is so relatively small that the electrode reaction tends to be more irreversible. $V$ is a dimensional parameter which represents the potential drops across the distance of the sphere's radius, and $\Psi$ is its dimensionless parameter.

So the governing equations and boundary conditions can be expressed in dimensionless form as follows

$$
\begin{aligned}
& \nabla^{2} \Phi_{1}=0 \\
& \nabla^{2} \Phi_{2}=0
\end{aligned}
$$

in which

$$
\nabla^{2}=\frac{1}{\xi^{2}} \frac{\partial}{\partial \xi}\left(\xi^{2} \frac{\partial}{\partial \xi}\right)+\frac{1}{\xi^{2} \sin \theta} \frac{\partial}{\partial \theta}\left(\sin \theta \frac{\partial}{\partial \theta}\right)
$$

The dimensionless boundary conditions are at $\xi=0$,

$$
\Phi_{1} \text { is finite }
$$

at $\xi \rightarrow \infty$,

$$
\Phi_{2}=-\xi \cos \theta+\Phi_{0}
$$

at $\xi=1$,

$$
\frac{\partial \Phi_{1}}{\partial \xi}=\alpha \frac{\partial \Phi_{2}}{\partial \xi}
$$

for a reversible reaction

at $\xi=1$,

$$
\Phi_{1}-\Phi_{2}=E
$$

for linear polarization

for $\xi=1$,

$$
\frac{\partial \Phi_{2}}{\partial \xi}=-\beta \Psi\left(\Phi_{1}-\Phi_{2}-E\right)
$$

for the Butler-Volmer kinetics 
at $\xi=1$,

$$
\begin{aligned}
\frac{\partial \Phi_{2}}{\partial \xi}=-\beta\left\{\operatorname { e x p } \left[\alpha _ { \mathrm { a } } \Psi \left(\Phi_{1}\right.\right.\right. & \left.\left.-\Phi_{2}-E\right)\right] \\
& \left.-\exp \left[-\alpha_{\mathrm{c}} \Psi\left(\Phi_{1}-\Phi_{2}-E\right)\right]\right\}
\end{aligned}
$$

The general solutions for Eq. [10] and [11] are

$$
\begin{aligned}
& \Phi_{1}=\sum_{n=0}^{\infty}\left[A_{n} \xi^{n} P_{n}(\cos \theta)+B_{n} \xi^{-(n+1)} P_{n}(\cos \theta)\right] \\
& \Phi_{2}=\sum_{n=0}^{\infty}\left[C_{n} \xi^{n} P_{n}(\cos \theta)+D_{n} \xi^{-(n+1)} P_{n}(\cos \theta)\right]
\end{aligned}
$$

which are the Zonal Harmonics (14). The function $P_{n}$ is a Legendre polynomial of degree $n$, and the coefficients $A_{n}$, $B_{n}, C_{n}$, and $D_{n}$ are to be determined according to the boundary conditions. Substituting Eq. [12]-[14] into Eq. $[16 a, b]$ gives the following equations

$$
\begin{gathered}
\Phi_{1}=\sum_{n=0}^{\infty} A_{n} \xi^{n} P_{n}(\cos \theta) \\
\Phi_{2}=\Phi_{0}-\xi \cos \theta+\sum_{n=1}^{\infty} D_{n} \xi^{-(n+1)} P_{n}(\cos \theta)
\end{gathered}
$$

in which $A_{0}$ and $\left\{D_{n}\right\}$ are unknown, and by use of current continuity, $\left\{A_{n}\right\}$ for $n \geq 1$ can be related to

$$
\begin{aligned}
& A_{1}=-\alpha\left(1+2 D_{1}\right) \\
& A_{n}=-\alpha\left(\frac{1+n}{n}\right) D_{n}, \quad \text { for } n \geq 2
\end{aligned}
$$

Then the solutions for various surface conditions can be obtained respectively from Eq. [17a, b] and Eq. [15a, b, c].

Analytical solution for linear polarization.-The potential distribution in each phase for the case of linear polarization can be obtained by substituting Eq. [15b] into Eq. $[17 \mathrm{a}, \mathrm{b}]$

$$
\begin{gathered}
\Phi_{1}=\Phi_{0}+E-\frac{3 \alpha}{1+2 \alpha+2 \gamma} \xi \cos \theta \\
\Phi_{2}=\Phi_{0}-\xi \cos \theta+\frac{1-\alpha-\gamma}{1+2 \alpha+2 \gamma} \frac{\cos \theta}{\xi^{2}}
\end{gathered}
$$

in which $\gamma=1 / \beta \Psi$. Equations [19a, b] are identical to those obtained by Maxwell (11), as the electrode reaction approaches complete reversibility, $\gamma \rightarrow 0$. It should be noticed that the sphere still has an influence on the potential distribution even in the case of $\alpha=1$, and this is caused by the resistance of the electrochemical reaction at the surface. The current distribution at the surface of the sphere for the linear polarized kinetics can be derived from the potential distribution and expressed as

$$
\frac{i_{n}}{i_{\infty}}=\frac{3}{1+2 \alpha+2 \gamma} \cos \theta
$$

Solutions for the Butler-Volmer kinetics.-The potential distributions for the two cases mentioned previously can be solved in analytical form. But, for the case of considering the Butler-Volmer kinetics, the coefficients $A_{0}$ and $D_{n}$ in Eq. [17a, b] and [18] may not be soluble analytically due to the nonlinearity of the boundary condition in Eq. [15c]. The boundary collocation method is suggested for obtaining the semianalytical solutions in this case. Substituting Eq. [17b] into Eq. [15c] gives

$$
\begin{aligned}
\sum_{n=1}^{\infty}(n+1) D_{n} P_{n}(\cos \theta)=\beta\left\{\exp \left[\alpha_{\mathrm{a}} \Psi\left(\Phi_{1}-\Phi_{2}-E\right)\right]\right. \\
\left.-\exp \left[-\alpha_{c} \Psi\left(\Phi_{1}-\Phi_{2}-E\right)\right]\right\}\left.\right|_{\xi=1}-\cos \theta
\end{aligned}
$$

By selecting $N$ points, $\left\{\theta_{\mathrm{i}}\right\}$, at the boundary to satisfy the above equation, a set of nonlinear algebraic equations for $D_{n}$ can be obtained and expressed as

$$
\begin{aligned}
\sum_{n=1}^{N-1}(n+1) D_{n} P_{n}\left(x_{\mathrm{i}}\right) & =\beta\left\{\operatorname { e x p } \left[\alpha_{\mathrm{a}} \Psi \eta\left(x_{\mathrm{i}}\right]\right.\right. \\
- & \left.\exp \left[-\alpha_{\mathrm{c}} \Psi \eta\left(x_{\mathrm{i}}\right)\right]\right\}-x_{\mathrm{i}} i=1, \ldots N
\end{aligned}
$$

in which $x_{\mathrm{i}}=\cos \theta_{\mathrm{i}}$, and

$$
\begin{aligned}
\eta\left(x_{\mathrm{i}}\right) & =\Phi_{1}-\Phi_{2}-E \\
& =\left(A_{0}-\Phi_{0}-E\right)+(1+\alpha) x_{\mathrm{i}}
\end{aligned}
$$$$
-\sum_{n=1}^{N-1}\left(1+\frac{n+1}{n} \alpha\right) D_{n} P_{n}\left(x_{\mathrm{i}}\right)
$$

where $\left(A_{0}-\Phi_{0}-E\right)$ in the above equation is regarded as an unknown in the calculation, and $A_{o}$ can be obtained after $\Phi_{0}$ and $E$ are specified. Therefore Eq. [22] can be solved numerically by the Newton-Raphson method, and the $N$ collocation points are chosen with equal division for $\theta$, in a range of 0 to $\pi$. Convergence is reached when $N$ is large enough to make the consecutive error less than $10^{-6}$. The current distribution at the surface of the sphere for the Butler-Volmer kinetics is given by

$$
\frac{i_{n}}{i_{\infty}}=\cos \theta+\sum_{n=1}^{N-1}(1+n) D_{n} P_{n}(\cos \theta)
$$

There $i_{n}$ can be calculated by Eq. [23] if the set of $D_{n}$ has been figured out by solving Eq. [22].

\section{Results and Discussion}

The solutions for all three cases satisfy the following integral constraint (13) automatically

$$
\iint_{\text {sphere }} i_{n} d A=0
$$

and thus the anodic and cathodic currents are balanced. In cited literature (5) the integral constraint is an extra condition, which has been used to determine the potential in the solid phase, where a uniform potential in the solid phase is assumed.

Current enhancement factor.-The current enhancement factor, $f_{\mathrm{E}}$, is defined as the ratio of the current passing through the sphere to the current passing through the same cross-section area without it. So it can be expressed as

$$
f_{\mathrm{E}}=\frac{I}{i_{\infty} \pi R^{2}}
$$

in which

$I=2 \pi \int_{\text {cathode }} i_{n} R^{2} \sin \theta d \theta\left(=2 \pi \int_{0}^{\pi / 2} i_{n} R^{2}\right.$

$\sin \theta d \theta$, for symmetric cases, $\left.\alpha_{\mathrm{a}}=\alpha_{\mathrm{c}}\right)$

When $f_{\mathrm{E}}>1$, the sphere is current attractive, i.e., the current passing through the sphere is increased due to its presence. The enhancement factor for a reversible reaction, $\gamma=0$, is given by Eq. [24] and [20] as

$$
f_{\mathrm{E}}=\frac{3}{1+2 \alpha}
$$

It is obvious that the maximum $f_{\mathrm{E}}$ is 3 , when $\alpha \rightarrow 0$. The enhancement factor $f_{\mathrm{E}}$ is smaller than 1 for $\alpha>1$. The sphere is regarded as an insulator as $f_{\mathrm{E}} \rightarrow 0$, for $\alpha \rightarrow \infty$. Without considering the reaction resistance, the current passing through the sphere is determined by the relative electric conductivity between the solid and electrolyte phases only. 
The current enhancement factor for linear polarized kinetics is also obtained by substituting Eq. [20] into [24]

$$
f_{\mathrm{E}}=\frac{3}{1+2 \alpha+2 \gamma} ; \quad \gamma=\frac{1}{\beta \Psi}
$$

Equation [26] is the same as Eq. [25] if $\gamma \rightarrow 0$, which corresponds to a reversible reaction. As can be seen in Eq. [26], the parameter $\gamma$ reduces the $f_{E}$ value, because the resistance of the interfacial electrode reaction has been involved in this case. The criterion for $f_{\mathrm{E}}>1$ is given from Eq. [26] by

$$
\gamma+\alpha<1
$$

in which $\gamma$ and $\alpha$ represent the relative resistances of the electrode reaction and electric conduction in the solid phase to electric conduction in the electrolyte phase, respectively. Because the electric current will choose a path with smaller resistance, the path through the sphere will be favored if the combined resistance of the electrode reaction and electric conduction in the sphere is smaller than that in the electrolyte.

The enhancement factor for Butler-Volmer kinetics can be calculated by the following equation, after $D_{n}$ values are obtained

$f_{\mathrm{E}}=2 \sum_{n=1}^{N-1} \frac{1+n}{1+2 n}$

$$
\left[P_{n-1}\left(x_{\mathrm{c}}\right)-P_{n+1}\left(x_{\mathrm{c}}\right)\right] D_{n}+1-x_{\mathrm{c}}^{2}
$$

where $x_{\mathrm{c}}=\cos \theta_{c}$, and $\theta_{c}$ is the angle of the interfacial position with $i_{n}=0$. $\theta_{c}$ is calculated by solving Eq. [23] with $i_{n}=0$. For symmetrical transfer coefficients $\alpha_{\mathrm{a}}$ and $\alpha_{\mathrm{c}}, \theta_{\mathrm{c}}$ is $90^{\circ}$, and then $x_{\mathrm{c}}=0$.

The enhancement factor for the Butler-Volmer kinetics is shown in the following figures. The effect of parameter $\Psi$ at various $\beta$ on the enhancement factor, $f_{E}$, with $\alpha=0$, is shown in Fig. 2. It is observed that $f_{\mathrm{E}}$ is an increasing function of $\Psi$, and a limiting value of $f_{\mathrm{E}}=3$ is obtained if $\Psi$ is sufficiently large. The maximum value of $f_{\mathrm{E}}$ is the same as that from the reversible reaction condition, Eq. [25]. Asymmetrical $\alpha_{\mathrm{a}}$ and $\alpha_{\mathrm{c}}$ will decrease $f_{\mathrm{E}}$, especially when $\beta$ is small. When $\beta$ is sufficiently large, the influence of $\alpha_{a}$ and $\alpha_{c}$ is diminished. In the case of symmetrical transfer coefficients, $\alpha_{\mathrm{a}}=\alpha_{\mathrm{c}}=0.5$, both anodic and cathodic reactions have the same resistance. But, if $\alpha_{c}$ is less than $\alpha_{a}$, the resistance of the cathodic reaction is increased so that $f_{E}$ is decreased. The parameter $\beta$ is the ratio of the exchange current density $i_{0}$ to the applied current density $i_{\infty}$; when $\beta$ is sufficiently large, the electrode reaction will approach linear polarized or reversible kinetics. In the case of linear po-

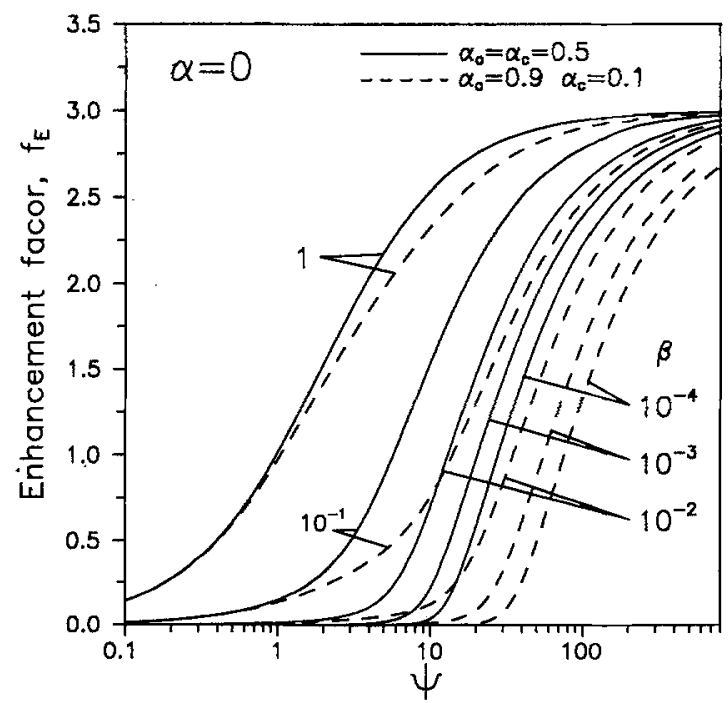

Fig. 2. The effects of $\Psi$ on the enhancement factor, $f_{\mathrm{Er}}$ at various $\beta$ with $\alpha=0$.

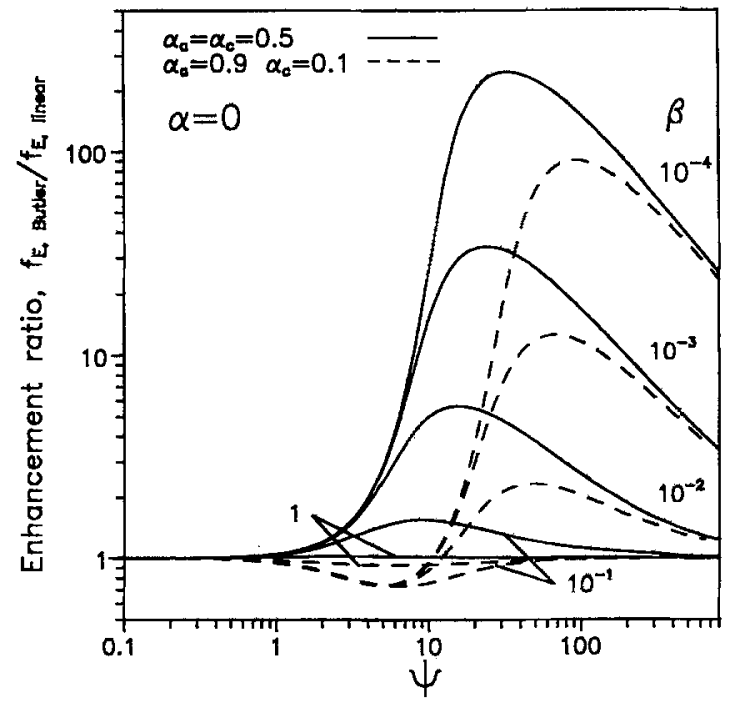

Fig. 3. The current enhancement factor ratio of the Butler-Volmer kinetics over the linear polarized kinetics.

larized kinetics, the effects of asymmetrical transfer coefficients will disappear, as seen in Eq. [15b].

A comparison of $f_{\mathrm{E}}$ between the Butler-Volmer and linear polarized kinetics at various $\beta$ and $\Psi$ is shown in Fig. 3 . When the enhancement ratio, $f_{\mathrm{E}, \mathrm{Butle}} / f_{\mathrm{E} \text {,linear, }}$ is equal to 1 , the same $f_{\mathrm{E}}$ value is predicted by either the Butler-Volmer kinetics or linear kinetics, and linear polarized kinetics is assumed reasonable in this situation. It is observed that the enhancement ratio is approximately equal to 1 for $\Psi<1$ over the whole range of $\beta$. When $\Psi$ is sufficiently large, the major resistance will be in the electrolyte so that the enhancement ratio of the Butler-Volmer kinetics over the linear kinetics is expected to approach 1 . There is a maximum of the enhancement ratio when $\Psi$ is in the range of 1-100. The enhancement ratio is smaller as $\beta$ is increased, and it is close to 1 for the whole range of $\Psi$ with $\beta=1$ as well as $\alpha_{\mathrm{a}}=\alpha_{\mathrm{c}}$. For asymmetrical transfer coefficients $\left(\alpha_{\mathrm{a}} \neq \alpha_{\mathrm{c}}\right)$, the enhancement ratio is apparently reduced and a minimum value is also observed while $\beta<10^{-2}$.

Current distribution on the spherical surface.-The current distribution on the sphere with $\alpha_{a}=\alpha_{c}$ is shown in Fig. 4. It is observed that higher $\Psi$ gives higher bipolar current, but the bipolar current is decreased as $\alpha$ increases. Nonuniform current distribution is obtained, and the current density passing through the sphere may be smaller than $i_{\infty}$ at a part of the surface, although the $f_{\mathrm{E}}$ is greater than 1 . The maximum current density occurs when $\theta$ is equal to 0 and $180^{\circ}$, and a zero current occurs at $\theta=90^{\circ}$. When $\beta=10^{-3}$, an inactive region appears around $\theta=90^{\circ}$. It should be noticed in Eq. [25] and [26] and Eq. [20] that the

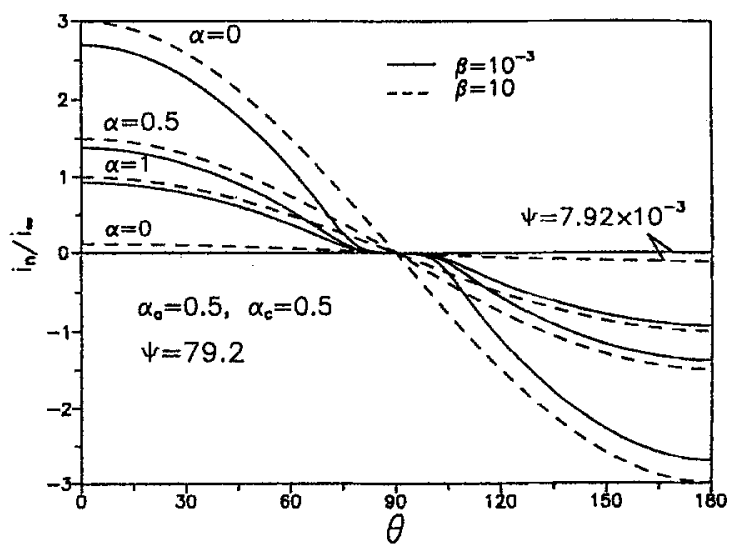

Fig. 4. Current distribution at the electrode surface for $\alpha_{a}=\alpha_{c}=0.5$ 


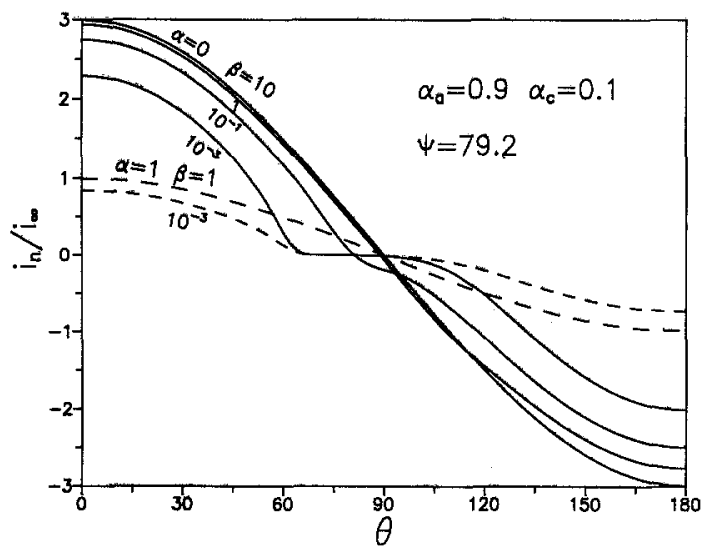

Fig. 5. The same as Fig. 4 except $\alpha_{a}=0.9$ and $\alpha_{c}=0.1$

magnitude of the maximum current density occurring at the surface is equal to its current enhancement factor, $f_{\mathrm{E}}$, and the current variation with $\theta$ is equal to multiplying $f_{\mathrm{E}}$ by $\cos \theta$ directly, for the reversible reaction and the linear kinetics, respectively. Almost no current passes through the sphere when $\Psi$ is sufficiently small. The current distribution on the sphere with asymmetrical transfer coefficients $\left(\alpha_{a} \neq \alpha_{c}\right)$ is shown in Fig. 5 . The asymmetrical current distribution is observed as $\beta$ is decreasing, and the area for the anodic reaction is smaller than for the cathodic reaction due to $\alpha_{\mathrm{a}}>\alpha_{\mathrm{c}}$. The inactive region still exists for $\beta=10^{-3}$, but it gradually disappears as $\beta$ is increasing. When $\beta$ is sufficiently large, a symmetrical distribution can also be observed, and the same results are obtained with $\beta<1$, whether $\alpha_{\mathrm{a}}$ and $\alpha_{\mathrm{c}}$ is symmetric or not.

Current distribution at the plane of $z=0$.--The current distribution at the plane of $z=0$ has been calculated and is shown in Fig. 6 . When $\xi \geq 2.5$, the presence of the spherical particle offers almost no influence. For the case of $\alpha=1$ and $\beta=10$, the particle has absolutely no effect on the electric field. This is because for the case of $\beta=10$ and $\Psi=79.2$, the resistance of the surface reaction can be neglected, and the resistance of electrical conduction in both the solid and the electrolyte phases is the same for $\alpha=1$. Thus, a linear electric field is still maintained and is not disturbed by the sphere. There are two typical kinds of curves. One is current-attractive, in which $f_{\mathrm{E}}$ is greater than 1 , and the current gets smaller the closer ones gets to the particle surface; the other one is current-repulsive, in which $f_{E}$ is less than 1 , and the current gets higher as one gets closer to the sphere. It is noticed that for smaller $\beta$, the sphere is more repulsive to current, owing to the increase of the resistance of the surface reaction. It is also observed specially in Fig. 6 that for $\alpha=0$ and $\beta=10^{-3}$, current density has been slightly enlarged near the spherical surface, although $f_{\mathrm{E}}$ is greater than 1 in this case. It can be explained by comparing with Fig. 4 that the inactive region

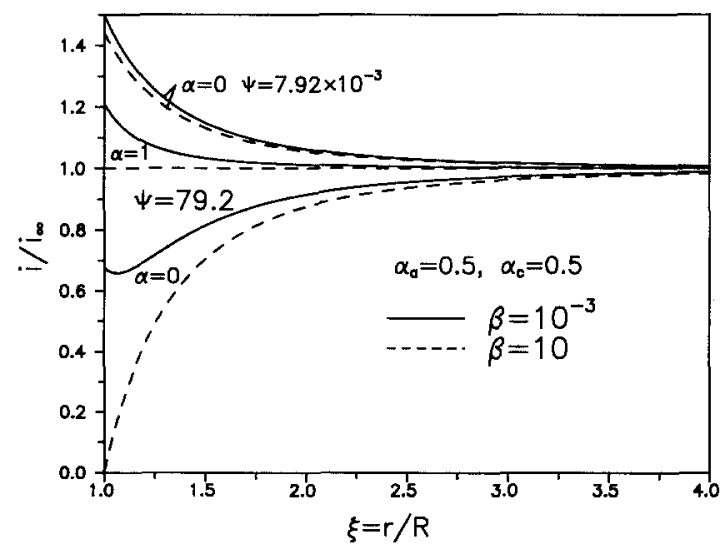

Fig. 6. Current distribution at the plane of $z=0\left(\theta=90^{\circ}\right)$ for $\alpha_{a}=\alpha_{c}=0.5$.

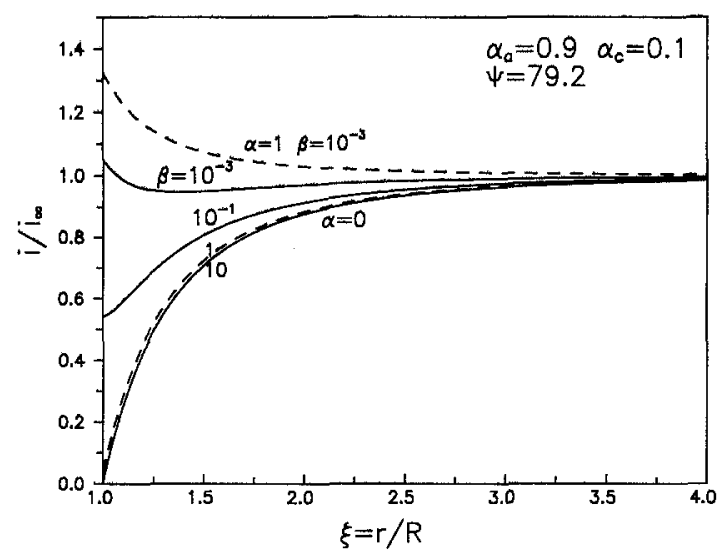

Fig. 7. The same as Fig. 6 except $\alpha_{a}=0.9$ and $\alpha_{c}=0.1$.

occurs near the top of the sphere $\left(\theta=90^{\circ}\right)$, and the current is to be repulsed near the inactive region, so that the current bypasses the sphere through the electrolyte phase. The current distribution at plane of $z=0$ with asymmetrical $\alpha_{\mathrm{a}}$ and $\alpha_{\mathrm{c}}$ is shown in Fig. 7. The effect of asymmetrical transfer coefficients is diminished as $\beta$ increases, due to the approach of linear polarized kinetics. Comparing it with the results from symmetrical transfer coefficients, the current passing through the plane of $z=0$ is higher, i.e., where more current is repulsed by the sphere owing to the higher reaction resistance produced by the asymmetrical transfer coefficients. As $\beta$ is decreased, the current near the electrode surface is increased; for $\alpha=1$, current near the sphere is always larger than $i_{\infty}$, whether $\alpha_{\mathrm{a}}$ and $\alpha_{\mathrm{c}}$ are symmetrical or not. It is also observed that when $\alpha=0$ and $\beta=10^{-3}$, the current becomes larger than $i_{\infty}$ near the sphere, although $f_{\mathrm{E}}$ is greater than 1 in this case. As mentioned, an inactive region observed at the electrode surface around $\theta=90^{\circ}$ may explain this phenomenon.

Effective conductivity of a dilute dispersion.-The difference of electric power consumption in the whole domain between the presence of a single sphere and its absence in a linear potential field is expressed as

$$
\begin{aligned}
Q= & \left\{\begin{array}{c}
\text { power consumption } \\
\text { inside the particle }
\end{array}\right\} \\
& +\left\{\begin{array}{c}
\text { power consumption } \\
\text { outside the particle }
\end{array}\right\}+\left\{\begin{array}{c}
\text { power consumption } \\
\text { at interface }
\end{array}\right\} \\
& -\left\{\begin{array}{c}
\text { power consumption in the whole domain } \\
\text { with the same current density }
\end{array}\right\}
\end{aligned}
$$

After rather complicated mathematical operations which include applying the Gauss divergence theorem and Green's theorem, the energy difference $Q$ can be related to the first coefficient $D_{1}$ of the series expression of the potential $\Phi_{2}$ in the continuous medium

$$
Q=\left(\frac{4 \pi R^{3}}{3 \kappa_{2}} i_{\infty}^{2}\right) \cdot D_{1}
$$

For linear polarization, according to Eq. [17b] and [19b], the energy difference $Q$ is

$$
Q=\left(\frac{4 \pi R^{3}}{3 \kappa_{2}} i_{\infty}^{2}\right) \cdot \frac{1-\alpha-\gamma)}{1+2 \alpha+2 \gamma}
$$

The effective conductivity of a dilute dispersion can be related to the bipolar analysis of a single sphere in a linear potential field by regarding the dispersion itself as an imaginary sphere of radius $S$ with an effective conductivity $\kappa_{e}$ in a continuous medium of conductivity $\kappa_{2}$ and a reversible electrochemical reaction at interface. By setting the energy consumption due to the presence of the imaginary sphere of radius $S$ in the same linear potential field 


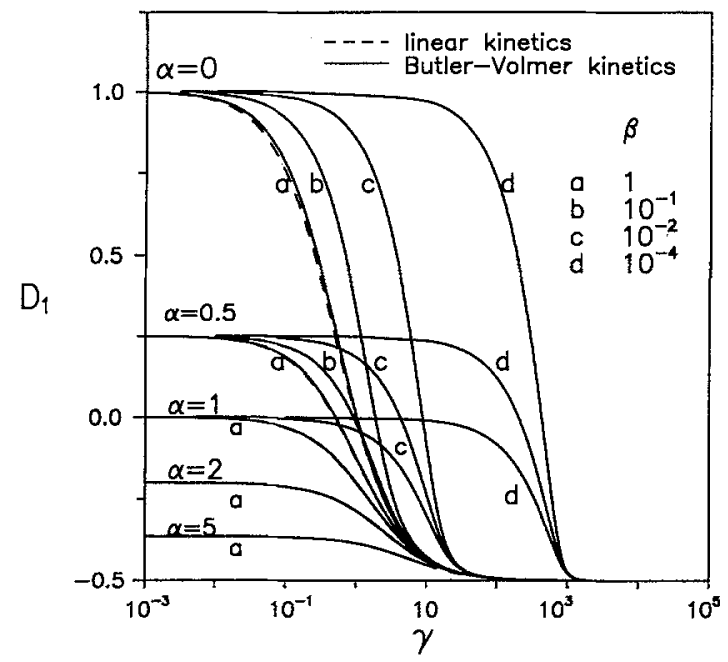

Fig. 8. The first coefficient $D_{1}$ vs. the kinetic parameter $\gamma$ for various $\alpha$ and $\beta$.

equal to that obtained due to the presence of the spherical particle of radius $R$ with conductivity $\kappa_{1}$, the following relation is obtained from Eq. [29]

$$
\left(\frac{4 \pi R^{3}}{3 \kappa_{2}} i_{\infty}^{2}\right) \cdot D_{1}=\left(\frac{4 \pi S^{3}}{3 \kappa_{2}} i_{\infty}^{2}\right) \cdot D_{1 \mathrm{e}}
$$

where

$$
D_{1 e}=\frac{1-\alpha_{e}}{1+2 \alpha_{e}} ; \quad \alpha_{e}=\frac{\kappa_{2}}{\kappa_{e}}
$$

Let $f$ be the volume fraction of the dispersed phase, then

$$
f=\left(\frac{R}{S}\right)^{3}
$$

By inserting Eq. [32] into Eq. [31] and using Eq. [33], the first coefficient $D_{1}$ can be related to the effective conductivity $\kappa_{e}$ by the following expression

$$
D_{1} f=D_{1 \mathrm{e}}=\frac{1-\alpha_{\mathrm{e}}}{1+2 \alpha_{\mathrm{e}}}=\frac{\kappa_{\mathrm{e}} / \kappa_{2}-1}{\kappa_{\mathrm{e}} / \kappa_{2}+2}
$$

Because the first coefficient $D_{1}$ of the series expression of the potential $\Phi_{2}$ is figured out analytically for linear polarization or numerically for Butler-Volmer kinetics, the effective conductivity can be computed with the known volume fraction $f$ of the dispersed phase. The first coefficient $D_{1} v s$. $\gamma$ for various $\alpha$ and $\beta$ is shown in Fig. 8. As shown in Fig. 8, two asymptotic values of $D_{1}$ are observed while $\gamma$ is approaching infinity as well as zero, respectively, in which the asymptotic $D_{1}$ can be calculated directly from the results of linear polarization. By rearranging Eq. [34], it can be concluded that the ratio $\kappa_{2} / \kappa_{2}$ is greater than 1 if $D_{1}>0$ and the presence of the dispersion is beneficial to electric conduction in the electrolyte phase. On the contrary, for $D_{1}<0, \kappa_{e} / \kappa_{2}$ is smaller than 1 , and the dispersion is an obstacle to electric conduction in the electrolyte phase.

For linear polarization, Eq. [34] becomes

$$
\left(\frac{1-\alpha-\gamma}{1+2 \alpha+2 \gamma}\right) f=\frac{\kappa_{\mathrm{e}} / \kappa_{2}-1}{\kappa_{\mathrm{e}} / \kappa_{2}+2}
$$

where $\alpha=\kappa_{2} / \kappa_{1}$ and $\gamma=1 / \beta \Psi$. As the electrochemical reaction approaches complete reversibility, $\gamma \rightarrow 0$; then Eq. [35] is reduced to

$$
\left(\frac{\kappa_{e} / \kappa_{2}-1}{\kappa_{\mathrm{e}} / \kappa_{2}+2}\right) f=\frac{\kappa_{\mathrm{e}} / \kappa_{2}-1}{\kappa_{\mathrm{e}} / \kappa_{2}+2}
$$

which is identical to that obtained by Maxwell $(11,12)$.

\section{Conclusion}

Analytical current and potential distributions have been obtained for a reversible reaction and a linear polarized kinetics, respectively. Semianalytical potential and current distributions for the Butler-Volmer kinetics are also obtained by the boundary collocation method. By comparing the results between the Butler-Volmer kinetics and the linear polarized kinetics or reversible reaction, it is concluded that the surface reaction can be regarded as showing linear polarized kinetics for $\beta \geq 1$, and the linear kinetics is a good assumption for $\beta<1$, if $\Psi<1$. When $\Psi$ is sufficiently large, the major resistance will be in the electrolyte so that the enhancement ratio of the Butler-Volmer kinetics over the linear kinetics will approach unity. For asymmetrical transfer coefficients $\left(\alpha_{\mathrm{a}} \neq \alpha_{\mathrm{c}}\right), f_{\mathrm{E}}$ is reduced, except that the linear polarized kinetics is approached. Nonuniform current distribution on the sphere is obtained, and its maximum value is at $\theta=0$ or $180^{\circ}$. For the linear polarized kinetics, the current variation with $\theta$ is the same as would be obtained by multiplying $f_{\mathrm{E}}$ by $\cos \theta \mathrm{di}$ rectly. For $\alpha_{a} \neq \alpha_{c}$, asymmetrical current distribution is obtained, and it becomes symmetrical when the linear polarized kinetics is approached. The potential field in the electrolyte is disturbed by the sphere within a distance of 2.5 times the sphere's radius. The effective conductivity of dilute dispersions has also been formulated from the results of the bipolar analysis of a single sphere in a linear potential field. It is related to the first coefficient $D_{1}$ of the series expression of the potential $\Phi_{2}$, which is obtained analytically for linear polarization and numerically for the Butler-Volmer kinetics.

\section{Acknowledgment}

This work was supported by the National Science Council, Taiwan, Republic of China, under Contract No. NSC790410-E002-60.

Manuscript submitted Dec. 3, 1990; revised manuscript received March 12, 1991.

National Taiwan University assisted in meeting the publication costs of this article.

\section{LIST OF SYMBOLS}

English characters
$A_{n}, B_{n}, C_{n}, D_{n}$
coefficients in Eq. [16a, b]
V
E
Nernst potential of a reversible reaction, $\mathrm{V}$
$f \quad$ as $E^{\prime} / V$ frame fraction of dispersion
Faraday constant, 96,500 C/equiv.
exchange current density, $\mathrm{A} / \mathrm{m}^{2}$
applied current density, $\mathrm{A} / \mathrm{m}^{2}$
local current density at the surface of the sphere, $\mathrm{A} / \mathrm{m}^{2}$
local Faraday current density, expressed by Eq. [7]
number of the collocation points selected number of charge transfer in an electro- chemical reaction
difference of power consumption arising from the presence of sphere, $\mathrm{J} / \mathrm{s}$ spatial variable in radial direction, $m$ radius of the sphere, $m$
gas constant
radius of an imaginary sphere, $m$ temperature, $\mathrm{K}$
potential drop across half the sphere, $i_{\infty} R / \kappa_{2}, \mathrm{~V}$
local position at the spheric surface, de-
$x_{\mathrm{i}} \quad \begin{aligned} & \text { local position at the spheric surface, de- } \\ & \text { fined as } \cos \theta_{\mathrm{i}}\end{aligned}$
$x_{\mathrm{c}} \quad$ the position where $i_{n}=0$ at the spheric surface, defined as $\cos \theta_{c}$
spatial variable in the axial direction, $m$
$z$

Greek characters

$\alpha \quad$ dimensionless parameter, $\kappa_{1} / \kappa_{2}$, defined by Eq. [9]

$\alpha_{a}, \alpha_{c} \quad$ anodic and cathodic transfer coefficients

$\alpha_{e} \quad$ dimensionless parameter, $\kappa_{e} / \kappa_{1}$ 
dimensionless parameter, $i_{0} / i_{\infty}$, defined by Eq. [9]

$\gamma \quad$ dimensionless parameter, $1 / \beta \Psi$

$\eta \quad$ dimensionless surface overpotential, $\Phi_{1}-\Phi_{2}-E$

$\theta$ spatial variable in the circumferential direction, radian

$\theta_{c} \quad$ the angle where $i_{n}=0$ at the spheric surface, radian

$\theta_{i}$ the angle of the boundary collocation points, radian

$\kappa_{1}$ electric conductivity in solid phase, $(\Omega \mathrm{m})^{-1}$

$\kappa_{2} \quad$ electric conductivity in electrolyte phase, $(\Omega \mathrm{m})^{-1}$

$\kappa_{\mathrm{e}} \quad$ effective electric conductivity, $(\Omega \mathrm{m})^{-1}$

$\xi \quad$ dimensionless radial position, $r / R$

$\phi_{1} \quad$ potential in solid phase, $V$

$\phi_{2}$

$\phi_{0}$ potential in electrolyte phase, $\mathrm{V}$ reference potential at the plane of $z=0$ far from the sphere, $\mathrm{V}$

$\Phi_{1}, \Phi_{2}, \Phi_{0} \quad$ dimensionless potential of $\phi_{1}, \phi_{2}, \phi_{0}$, defined by Eq. [8]

$\Psi$

dimensionless parameter defined by Eq. [9]

\section{REFERENCES}

1. M. A. Hartland and A. J. M. Spencer, Trans. Inst. Chem. Eng., 41, 328 (1963).
2. P. R. Nadebaum and T. Z. Fahidy, Electrochim. Acta, 20, 715 (1975).

3. C. J. H. King, K. Lister, and R. E. Plimley, Trans. Inst. Chem. Eng., 53, 20 (1975).

4. F. Goodridge, C. J. H. King, and A. R. Wright, Electrochim. Acta, 22, 347 (1977).

5. R. Alkire, This Journal, 120, 900 (1973).

6. K. Kusakable, S. Morooka, and Y. Kato, J. Chem. Eng. Jpn. 15, 45 (1982).

7. B. J. Sabacky and J. W. Evans, Metall. Trans., 8B, 5 (1977).

8. J. K. Lee, L. W. Shemilt, and H. S. Chun, J. Appl. Electrochem., 19, 877 (1989).

9. D. C. Eardley, D. Handley, and S. P. S. Andrew, Electrochim. Acta, 18, 839 (1973).

10. K. Kusakabe, S. Morooka, and Y. Kato, J. Chem. Eng. Jpn., 19, 43 (1986).

11. J. C. Maxwell, "Electricity and Magnetism," Vol. 1, 3rd ed., p. 8, Oxford (1892).

12. R. E. Meredith and C. W. Tobias, "Advances in Electrochemistry and Electrochemical Engineering," Vol. 2, Chap. 2, John Wiley and Sons, Inc., New York (1962).

13. M. J. Gluckman, R. Pfeffer, and S. Weinbaum, J. Fluid Mech., 50, 705 (1971).

14. P. M. Morse and H. Feshbach, "Methods of Theoretical Physics," p. 124, McGraw-Hill Co., New York (1978).

15. P. S. Fedkiw and M. A. Bogard, This Journal, 135, 1726 (1988).

\title{
Experimental Approach to the Impedance Spectroscopy Technique
}

\author{
G. Chiodelli \\ C.S.T.E.-C.N.R., Dipartimento di Chimica Fisica, Universitá di Pavia, 27100 Pavia, Italy
}

P. Lupotto

S.E.A. Laboratoria, 13020 Pila, VC, Italy

\begin{abstract}
The performances of commercially available frequency response analyzers, currently used in electrochemical impedance spectroscopy, have been improved by two methods. The first approach concerns the management of the data acquisition and correction by means of an interactive graphic program. Moreover, a new software is described, allowing the expansion by two orders of magnitude the impedance range of the instrument. The second way consists in a homemade, high-impedance adaptor $\left(10^{12} \Omega, 3 \mathrm{pF}\right)$ with a guard circuit driver, that increases the sensitivity of the analyzer. Thus, the electrical behavior of materials with high impedance modulus can be investigated.
\end{abstract}

Among the various experimental techniques currently adopted in electrochemistry, impedance spectroscopy (IS) has proved to be a valuable research tool thanks to its sensitivity and to the wide variety of possible applications, as it was foreseen by Bauerle (1) in his early work.

Three classes of instruments are principally used in electrochemical impedance spectroscopy: multifrequency bridges, lock-in amplifiers, and frequency response analyzers (FRA).

In recent years, great efforts were devoted to data analysis, to conversion of impedance plots in meaningful information through suitable models of equivalent circuits $(2,3)$, to parameter extractions with nonlinear least squares fit (NLLF) programs $(4,5)$, and to graphic displays with different formalisms (6). In particular, availability of more powerful personal computers has allowed the design of new software for routine analysis of data $(4,5,7)$.

On the other side, there is a great need of expanding the input impedance of the commercial apparatus by means of an improved hardware system which can be defined as an "impedance adaptor" (7-9). An interesting goal of this possible development is the characterization of low conductivity samples, viz. ceramic oxides based electrolytes (10-13) at temperatures lower than $300^{\circ} \mathrm{C}$.
In this paper, a software program that manages the data acquisition and the interactive graphic display in real time is presented. In the section on Data acquisition, treatment, and graphic display a flow-chart diagram will be discussed. Several routines for the correction of data from the parasitic element effects are also illustrated. Moreover, a new device, the above mentioned impedance adaptor is described, and its circuit and applications in different fields are shown.

\section{Experimental}

Theory of the measurements.-A perturbating sinusoidal signal $E=E_{0} \sin (\omega t)$ at frequency $F(\omega=2 \pi F)$ is applied to the unknown sample, and the response is analyzed by a correlator in terms of the resulting current $I=I_{0}$ sin $(\omega t+\varphi)$, where $\varphi$ represents the phase angle shift.

The corresponding complex impedance, obtained by varying the signal pulsation $\omega$, is expressed by the displacement of the vector $\overrightarrow{\mathbf{Z}}(\omega)$ with its parameters, the modulus $Z_{0}=E_{0} / I_{0}$ and the phase angle $\varphi$ (polar coordinates) or with the real and imaginary values of the impedance (cartesian coordinates), as shown in Fig. 1.

As an example, in Fig. 2 a simple equivalent circuit of an electrochemical cell, a resistance in parallel with a capaci- 